\section{ARS Tech}

Authors retain copyright

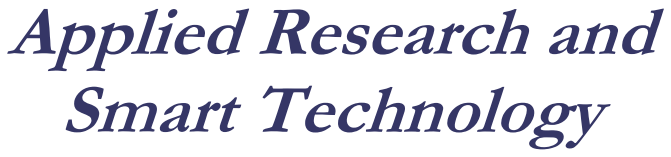

Journal homepage: www.journals2.ums.ac.id/index.php/arstech

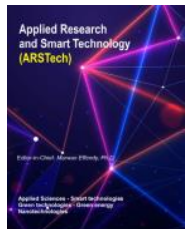

ISSN 2722-9645

Research Article

\title{
Low-cost pick and place anthropomorphic robotic arm for the disabled and humanoid applications
}

\author{
Norsinnira Zainul Azlan ${ }^{1^{*}}$, Mubeenah Titilola Sanni ${ }^{2}$, Ifrah Shahdad ${ }^{3}$ \\ ${ }^{1,2,3}$ Department of Mechatronics Engineering, Kulliyyah of Engineering, International Islamic University Malaysia, \\ Jalan Gombak, 53100 Kuala Lumpur, Malaysia.
}

*Corresponding author: sinnira@iium.edu.my

Permalink (DOI): https://doi.org/10.23917/arstech.v1i2.25

\begin{tabular}{|c|c|}
\hline ARTICLE INFO & ABSTRACT \\
\hline Article history: & This paper presents the design and development of a new low-cost pick and place \\
\hline Received 01 May 2020 & anthropomorphic robotic arm for the disabled and humanoid applications. \\
\hline Revised 05 Aug 2020 & Anthropomorphic robotic arms are weapons similar in scale, appearance, and \\
\hline Kevised Us Aug 2020 & functionality to humans, and functionality. The developed robotic arm was \\
\hline Accepted 03 Uct 2020 & simple, lightweight, and has four degrees of freedom (DOF) at the hand, \\
\hline $\begin{array}{l}\text { Avallable online } 30 \text { Nov } 2020 \\
\text { Published regularly } 15 \text { Dec } 2020\end{array}$ & shoulder, and elbow joints. The measurement of the link was made close to the \\
\hline 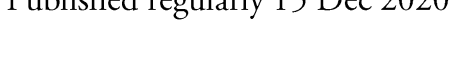 & $\begin{array}{l}\text { length of the human arm. The anthropomorphic robotic arm was actuated by } \\
\text { four DC servo motors and controlled using an Arduino UNO microcontroller }\end{array}$ \\
\hline Keywords: & board. The voice recognition unit drove the command input for the targeted \\
\hline thropomorphic robot & object. The forward and inverse kinematics of the proposed new robotic arm has \\
\hline Kinematics analysis & been analysed and used to program the low-cost anthropomorphic robotic arm \\
\hline Low-cost robot & prototype to reach the desired position in the pick and place operation. This \\
\hline Pick and place & paper's contribution is in developing the low cost, light, and straightforward \\
\hline Robotic arm & weight anthropomorphic arm that can be easily attached to other applications \\
\hline & such as a wheelchair and the kinematic study of the specific robot. The low-cost \\
\hline & robotic arm's capability has been tested, and the experimental results indicated \\
\hline & $\begin{array}{l}\text { that it could perform basic pick place tasks for the disabled and humanoid } \\
\text { applications. }\end{array}$ \\
\hline
\end{tabular}

\section{INTRODUCTION}

Robotic arms or manipulators have been an interesting topic for numerous researchers worldwide for many years. In the early days, robotic arms are initially designed to handle dangerous objects such as radioactive materials and to perform tasks in areas that are difficult to be reached [1]. As the field's interest has expanded over time, more robotic arms have been developed to perform repetitive and precise operations in various applications such as industrial, medical, and space exploration. In the industry, robotic arms are used for welding, materials handling, machine tending, component assembling, device picking, and product packing. In these applications, 
the mechanical arm design's primary consideration is the robots' functional capacity to complete the programmed tasks effectively. The appearance or artistic aspect of imitating a human arm is not vital in the robot design. However, with the growth of social robots' interest, humanoids, prosthetics limbs, assistive robots, and household robots' fields in recent decades, more attention has been given to developing robotic arms with anthropomorphic features.

An anthropomorphic robotic arm resembles a human arm's physical appearance, weight, and functionality [1] - [3]. This arm is mainly programmed to perform similar tasks as the human arm in a daily living setting. It is primarily used for prosthetics or artificial limbs applications to assist the disabled or amputees who had lost their arms due to accidents or illness. The arm alleviates their difficulties and reduces their dependency on the caregiver, which allows for independent living and selfcare. Generally, an anthropomorphic arm is preferred over the industrial-look robotic arm since it looks more natural and closer to the disabled's original hands. Anthropomorphic arms are also used in humanoid applications where the design needs to emulate a human's arm closely. However, the design and development of an anthropomorphic arm are highly challenging. Mimicking a human arm, including the hand, with a total of 34 degrees of freedom (DOF) while maintaining the human arm's original functionality, size and weight, while keeping the design cost low is not an easy task. An anthropomorphic robotic design usually requires complex joint mechanisms, including gimbals, hinges, linkages, springs, belts, and gears, which may incur a high cost to realise the kinematic mechanism and functionality of a human hand [1].

Previous works on anthropomorphic arms [4]-[7] include the study by Bandara et al. where the researchers developed a multi-DOF trans humeral robotic arm prosthesis, combining eight passive joints and seven active joints to realise a 15 DOF motion. The wrist is developed based on a parallel manipulator-based mechanism to attain a two-axis movement [7]. Chen et al. has coupled all motors with wires to design a 7 DOF anthropomorphic robotic arm to achieve high torque to weight ratio. The safety and durability issues due to wire characteristics have been considered in the construction [8]. The 3D printed materials and low-cost servo motors have been selected to develop a Raspberry Pi board, the I2C protocol-driven anthropomorphic arm, to reduce the design complexity. The material is flexible and light in weight. However, it produces undesirable oscillations that cause instability in the robotic arm performance. The chosen servomotors also give a smaller amount of torque than required, making it difficult for the robot to move the joints with heavy loads, especially the joint at the lower base [9]. In the 31 inches, 7 pounds and 5 DOF robotic arm developed by Shen et al., the 3D-printed components also have been chosen to make up mechanical arm structures using nylon and polylactic acid linked to carbon fibre tubing's. The distal and proximal joints are actuated by two servo motors and three brushless DC motors, respectively. Harmonic gearboxes are assembled at the joints to obtain zero backlashes [10]. Pop and Stan use the 3D human hand position and orientation data from the computer vision and pass it to a 6 DOF robotic arm so that the robot can move naturally, mimicking a human arm [11]. In the 23 DOF bionic robotic arm developed by Liao et al., the human arm movement is imitated using the operator's arm colour, depth image, and bones data obtained from Kinect [12].

An anthropomorphic robotic arm can be driven in many ways, including arm sensory glove and arm sleeve, vision system, Electromyography (EMG) signals, and brain-computer interfaces (BCIs). Oliveira et al. developed a 10 DOF anthropomorphic robotic arm using ten servo motors as actuators and driven by six flex sensors and two Inertial Measurement Units (IMU) to replicate human arm movements [13]. Grasshoff et al. used the multiple depth cameras matched to one another through a $3 \mathrm{D}$ calibration procedure in moving a 7 DOF anthropomorphic robotic arm to track human arm and hand frequencies [14]. Athanasiou et al. studied anthropomorphic robotic arms regulated by the off-theshelf BCI for assistive technologies and rehabilitation applications [15]-[17]. The BCI perceives the brain activity signals and interprets the data so that humans can move any robotics equipment attached to them without moving any parts of their body. Even though good results and anthropomorphic structures are achievable in these designs, most of these robotic arms are complicated and require high cost.

The study proposes a simple, lightweight, and lowcost anthropomorphic robotic arm, specifically for pickand-place tasks, an essential task in a prosthetics or humanoid application. This paper's contribution is in the low-cost anthropomorphic automated hand design and its specific kinematic analysis. This paper is organised as follows. The robotic arm's design is explained below, followed by the kinematics analysis and results and discussion sections. Finally, the conclusion of the study is drawn in the last quarter of the paper.

\section{DESIGN OF THE ROBO'TIC ARM}

The project research developed a low-cost and straightforward robotic hand, where the links' measurement was close to a human arm's size. The critical joints to realise pick and place task done by humans were identified as the 2 DOF shoulder joint and 1 DOF elbow joint, making $3 \mathrm{DOF}$ on the arm and $1 \mathrm{DOF}$ for the hand. 
From the several tests that have been done, it has been observed that the four identified joints were sufficient to accomplish the desired task. Therefore, the wrist was not actuated to save the development cost and simplify the design. A ready-made lightweight, flexible robotic hand was installed at the robot's wrist for the end effector. The robotic hands' five fingers were attached to a servo motor mounted at the wrist joint using strings to drive the hand in realising object grasping and releasing motions. All joints were designed to allow the movement of both clockwise and counter clockwise. The arm needed to be as light as possible to maximise the amount of the payload that it could carry, and the material for the arm structure required to be strong and rigid. Therefore, aluminium has been chosen to fulfil this requirement. The mechanical design of the low-cost robotic arm is displayed in Figure 1.

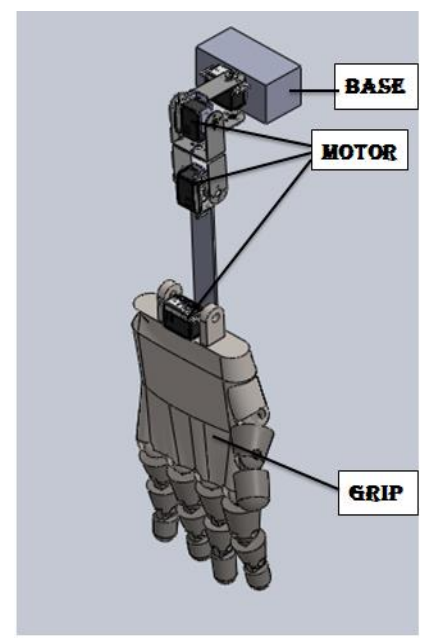

Figure 1. Mechanical design of anthropomorphic robotic arm (isometric view).

DC servo motors (model number STD-TS 53) have been chosen to actuate the robotic arm. The motor contains a brushed motor coupled to a sensor, which allows feedback control. Two DC servo motors were installed at the shoulder joint, one DC motor was mounted at the elbow joint, and one more DC motor was fixed at the wrist for hand opening and closing. DC servo motors have been chosen for the actuation since they were low in cost and provide the required torque for the anthropomorphic arm. An Arduino Microcontroller Board was selected as the controller unit in controlling the whole system since it was popular, relatively cheap, and easy to use. USB cable was used to connect the board for sending and receiving the serial data. The electrical circuit consisted of hooking each servo motor up to a ground, power, and signal wire. All the ground wires were combined and connected to the battery's black (-) wire and one of the ground inputs on the Arduino Microcontroller Board. The power lines were linked to the red $(+)$ wire of the battery, and the signal wires were connected to digital pins: $3,5,6$, and 9 .
The project used a voice recognition shield called the EasyVR module to receive verbal commands and enable the robotic arm to be more user friendly and easy to use. It consisted of microphone input, a speaker output, and audio line-out/headphone jack components and was simply connected to the controller by mounting it on top of the Arduino Microcontroller board. In the study, the voice recognition was programmed such that the robotic arm can pick two items: the food and glass, under the respective "FOOD" and "GLASS" command words. When either of these commands was spoken to the microphone, the EasyVR shield recognised the voice and captured the audio. The audio was then matched with the one existing in the database. The motors were then activated to move the robotic arm to the respective object position as instructed by the operator. Figure 2 shows the flow chart of the voice recognition.

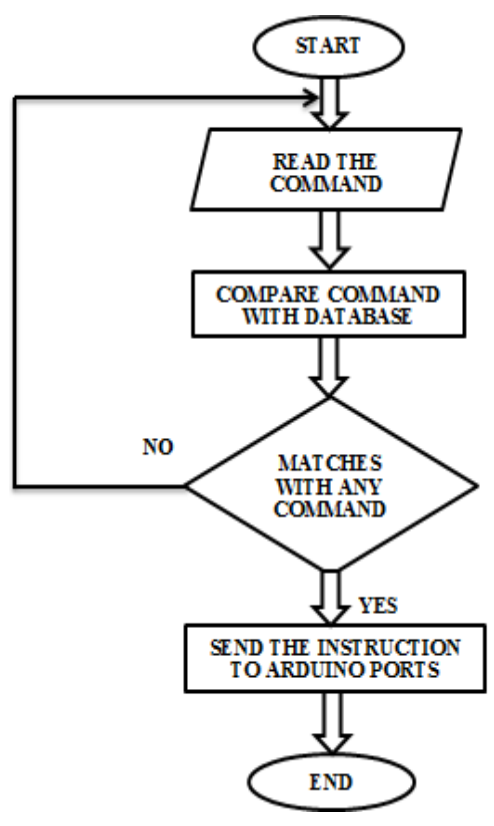

Figure 2. Flowchart for voice recognition.

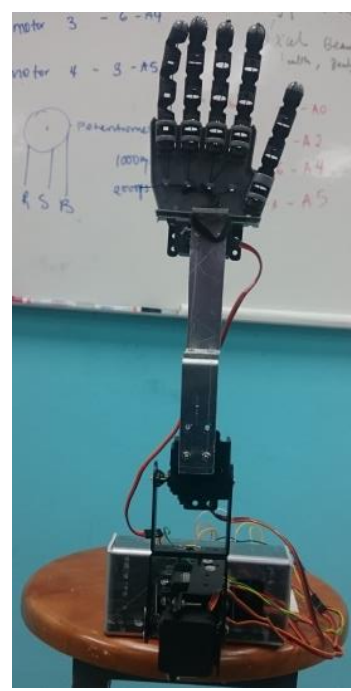

Figure 3. An anthropomorphic robotic arm. 
An emergency button was equipped in the hardware construction so that the robotic arm stopped its whole operation once the button was pressed. It served as a safety precaution since there was a risk that the robot might inflict injuries on the operator or the robot itself due to malfunction or any programming errors. The cost incurred for the robotic arm design was RM700. The complete low-cost anthropomorphic robotic arm is illustrated in Figure 3.

Figure 4 describes the operational sequence of the low-cost anthropomorphic robotic arm in picking and placing objects. Initially, the voice recognition would identify the type of chosen object. At this stage of the study, the object's locations were stored a priori in the robot's database. The robot accepted the command, and from the inverse kinematics equations, the amount of angle that each joint need to rotate was provided to the motors. The motors were then activated based on the given amount of angle they needed to turn by the microcontroller.

Once the desired position was achieved, the motors at the wrist would be activated so that the hand would grasp the object. Next, the shoulder and elbow moved the object to the desired destination, again using the inverse kinematics analysis. Lastly, the hand released the object once the final goal was reached and returned to its home position. The forward-and-inverse kinematics analysis of the developed anthropomorphic robotic arm needed in programming would be provided in the next section.

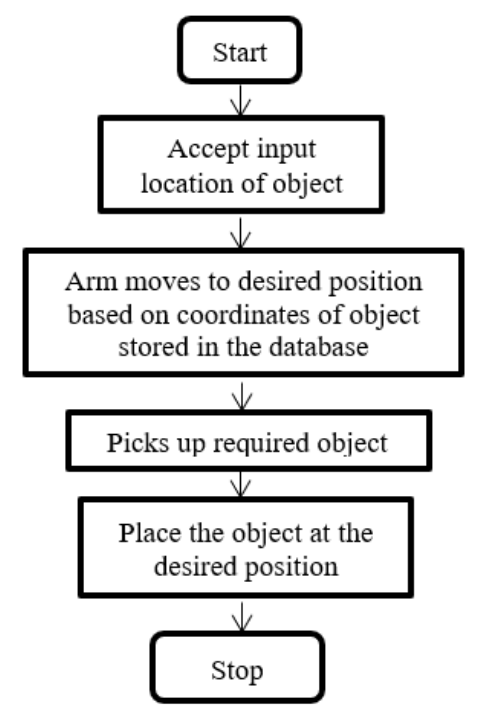

Figure 4. Flowchart of object pick-up.

\section{KINEMATICS ANALYSIS}

The analysis of the forward and inverse kinematics of a mechanical structure is vital in robotics research to examine the system's behaviour, whether it can function and perform as per design requirement. Kinematics analysis involves examining objects' movement without considering the forces or moments that lead to the motion [18]-[19]. Inverse kinematic equations are necessary to determine the joint's angular displacement for each joint required to position the low-cost anthropomorphic robotic hand at the object's original position and its final destination [20]-[23]. However, before obtaining this equation, the forward kinematics analysis, which involves the computation of the robotic hand position in Cartesian space concerning the provided joint angles, need to be conducted first.

The $\mathrm{Z}$ and $\mathrm{X}$-axis of each robotic arm joint have been assigned using the Denavit-Hartenberg (DH) convention, as illustrated in Figure 5. The DH parameters obtained are listed in Table 1.

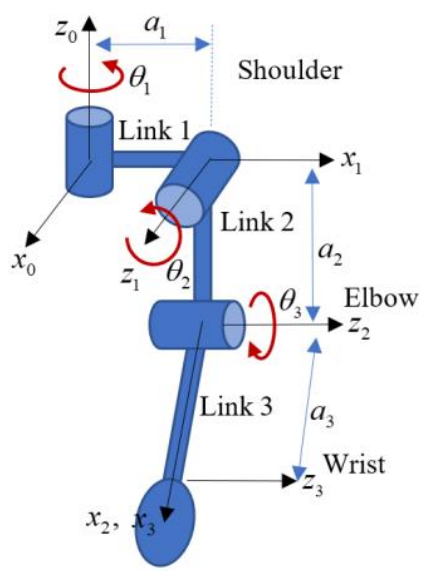

Figure 5. Kinematic model of the arm.

Table 1: DH parameters for the robot arm.

\begin{tabular}{ccccc}
\hline Link & $\theta$ & $d(\mathbf{m})$ & $\boldsymbol{a}(\mathrm{m})$ & $\alpha$ \\
\hline 1 & $\theta_{1}$ & 0 & $a_{1}$ & $90^{\circ}$ \\
2 & $\theta_{2}$ & 0 & $a_{2}$ & $-90^{\circ}$ \\
3 & $\theta_{3}$ & 0 & $a_{3}$ & $0^{\circ}$ \\
\hline
\end{tabular}

Forward kinematics analysis provides the position and the orientation of the low-cost anthropomorphic robotic arm's end-effector concerning the given joint angles at the shoulder and elbow. The forward kinematics could be calculated using the known link length and joint angles. Since the robotic arm has three degrees of freedom between the elbow and wrist, three matrix equations describe each link's orientation and position. From Table 1 , the forward kinematics of the anthropomorphic robotic arm, $A_{n}$ for $n$th link can be obtained as:

$$
A_{1}=\left[\begin{array}{cccc}
\cos \theta_{1} & 0 & \sin \theta_{1} & a_{1} \cos \theta \\
\sin \theta_{1} & 0 & -\cos \theta_{1} & a_{1} \sin \theta_{1} \\
0 & 1 & 0 & 0 \\
0 & 0 & 0 & 1
\end{array}\right]
$$


$A_{2}=\left[\begin{array}{cccc}\cos \theta_{2} & 0 & -\sin \theta_{2} & a_{2} \cos \theta_{2} \\ \sin \theta_{2} & 0 & \cos \theta_{2} & a_{2} \sin \theta_{2} \\ 0 & -1 & 0 & 0 \\ 0 & 0 & 0 & 1\end{array}\right]$

$A_{3}=\left[\begin{array}{cccc}\cos \theta_{3} & -\sin \theta_{3} & 0 & a_{3} \cos \theta_{3} \\ \sin \theta_{3} & \cos \theta_{3} & 0 & a_{3} \sin \theta_{3} \\ 0 & 0 & 1 & 0 \\ 0 & 0 & 0 & 1\end{array}\right]$

Multiplying equations (1)-(3) gives a (4) by four transformation matrices, where

$$
\begin{gathered}
A_{1} A_{2} A_{3}=\left[\begin{array}{c}
\cos \theta_{1} \cos \theta_{2} \cos \theta_{3}-\sin \theta_{1} \sin \theta_{3} \\
\sin \theta_{1} \cos \theta_{2} \cos \theta_{3}+\cos \theta_{1} \sin \theta_{3} \\
\sin \theta_{2} \cos \theta_{3} \\
0
\end{array}\right. \\
-\cos \theta_{1} \cos \theta_{2} \sin \theta_{3}-\sin \theta_{1} \cos \theta_{3} \\
-\sin \theta_{1} \cos \theta_{2} \sin \theta_{3}+\cos \theta_{1} \cos \theta_{3} \\
-\sin \theta_{2} \sin \theta_{3} \\
0 \\
-\cos \theta_{1} \sin \theta_{2} \\
-\sin \theta_{1} \sin \theta_{2} \\
\cos \theta_{2} \\
0
\end{gathered}
$$

$a_{3} \cos \theta_{1} \cos \theta_{2} \cos \theta_{3}+a_{2} \cos \theta_{1} \cos \theta_{2}-a_{3} \sin \theta_{1} \sin \theta_{3}+a_{1} \cos \theta_{1}$ $a_{3} \sin \theta_{1} \sin \theta_{2} \cos \theta_{3}+a_{2} \sin \theta_{1} \sin \theta_{2}+a_{3} \cos \theta_{1} \sin \theta_{3}+a_{1} \sin \theta$ $a_{3} \sin \theta_{2} \cos \theta_{3}+a_{2} \sin \theta_{2}$

The product of the equation (1) - (3) or equation (4) can be denoted as

$A_{1} A_{2} A_{3}=\left[\begin{array}{cccc}N_{x} & O_{x} & A_{x} & P_{x} \\ N_{y} & O_{y} & A_{y} & P_{y} \\ N_{z} & O_{z} & A_{z} & P_{z} \\ 0 & 0 & 0 & 1\end{array}\right]$

From equations (4) - (5), the inverse kinematics equation to determine the amount of the joint angles that the microcontroller needs to command the motor for each joint can be obtained. Equating equation (4) to equation (5), the elements of the matrices can be rewritten as

$N_{x}=\cos \theta_{1} \cos \theta_{2} \cos \theta_{3}-\sin \theta_{1} \sin \theta_{3}$

$N_{y}=\sin \theta_{1} \cos \theta_{2} \cos \theta_{3}+\cos \theta_{1} \sin \theta_{3}$

$N_{z}=\sin \theta_{2} \cos \theta_{3}$

$O_{x}=-\cos \theta_{1} \cos \theta_{2} \sin \theta_{3}-\sin \theta_{1} \cos \theta_{3}$

$O_{y}=-\sin \theta_{1} \cos \theta_{2} \sin \theta_{3}+\cos \theta_{1} \cos \theta_{3}$

$O_{z}=-\sin \theta_{2} \sin \theta_{3}$
$A_{x}=-\cos \theta_{1} \sin \theta_{2}$

$A_{y}=-\sin \theta_{1} \sin \theta_{2}$

$A_{z}=\cos \theta_{2}$

$P_{x}=a_{3} \cos \theta_{1} \cos \theta_{2} \cos \theta_{3}+a_{2} \cos \theta_{1} \cos \theta_{2}$

$-a_{3} \sin \theta_{1} \sin \theta_{3}+a_{1} \cos \theta_{1}$

$P_{y}=a_{3} \sin \theta_{1} \cos \theta_{2} \cos \theta_{3}+a_{2} \sin \theta_{1} \cos \theta_{2}$ $+a_{3} \cos \theta_{1} \sin \theta_{3}+a_{1} \sin \theta_{1}$

$P_{z}=a_{3} \sin \theta_{2} \cos \theta_{3}+a_{2} \sin \theta_{2}$

From equation (6) and rearranging for $A_{z}$, the angular displacement for link 2, $\theta_{2}$ can be determined as

$\theta_{2}=\cos ^{-1}\left(A_{z}\right)$

From the expression for $P_{Z}$ in equation (6), the joint angle for link 3 at the elbow can be calculated as

$\theta_{3}=\cos ^{-1}\left(\frac{P_{z}-a_{2} \sin \theta_{2}}{a_{3} \sin \theta_{2}}\right)$

The value of $\theta_{1}$ to drive the robotic hand to the desired position can be obtained from $A_{x}$

$$
\theta_{1}=\cos ^{-1}\left(\frac{-A_{x}}{\sin \theta_{2}}\right)
$$

Therefore, provided that Cartesian coordinates of the initial position or final destination object are available, such as from the camera, the robot joints' angular displacements or the amount that the motor needs to rotate can be calculated using equations (7) - (9).

\section{RESULTS AND DISCUSSION}

In the experimental test, the proposed low-cost anthropomorphic robotic arm has been tested to pick up food and glass and place the objects on a chair. In the experiment, the coordinates of the location of the food and the glass, as well as the destination of these two items, were assumed to be known and pre-programmed onto the Arduino Microcontroller board. The microcontroller then calculates each motor's respective joint angles to turn using the inverse kinematics in equations (7) - (9). Two scenarios consisting of picking up the food and glass to the designated location have been conducted, and the test has been repeated ten times. The experiment worked, it can be observed that the low-cost robotic arm has successfully achieved the desired pick and place task. The anthropomorphic robotic arm's movement in picking up and placing the objects is illustrated in Figure 6. 


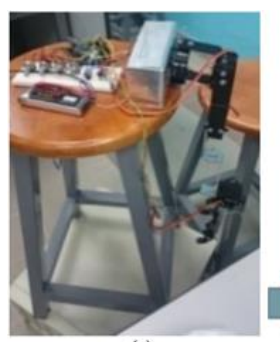

(a)

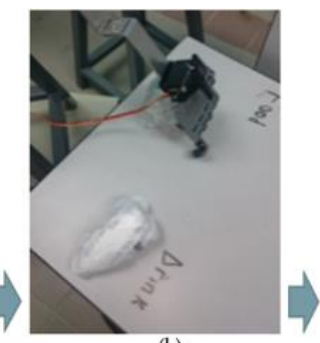

(b)

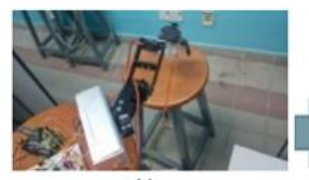

(c)

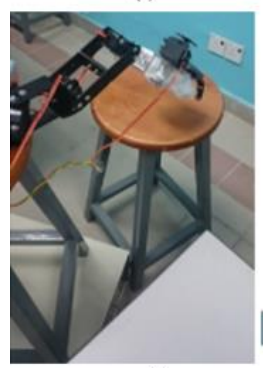

(e)

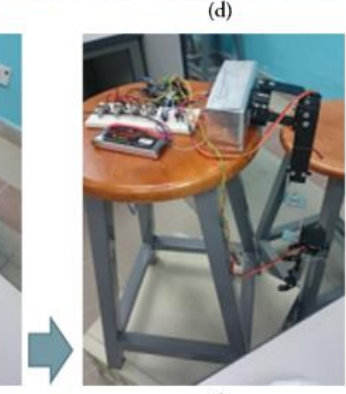

(f)
Figure 6. The sequence of movements of the proposed low cost anthropomorphic robotic while picking and placing food and glass. Anthropomorphic robotic hand (a) at home position, (b) picks up food, (c) places food at the desired position, (d) picks up the glass, (e) places the glass at the desired position, and $(f)$ returns to the home position.

The robotic arm starts the operation from the home position. The food has been picked up and placed in the required destination. Next, the glass has been successfully collected and placed at the right destination. Finally, the low-cost robotic arm has successfully returned to the home position. Table 2 shows the resulting angular displacements for each robot joint in degrees to reach the food, glass, final destination, and home positions. $\theta_{1}$ and $\theta_{2}$ represent the angle of rotation for the shoulder servomotors, while $\theta_{3}$ represents the displacement realised by the elbow servomotor and $\theta_{4}$ denotes the angle at which the servo mounted at the wrist turns for opening and closing the robot's hand to grasp and release objects.

Table 2. Motor angles.

\begin{tabular}{ccccc}
\hline & $\theta_{1}$ & $\theta_{2}$ & $\theta_{3}$ & $\theta_{4}$ \\
\hline Home Position & 90 & 0 & 90 & 180 \\
Food & 50 & 100 & 120 & 0 \\
Glass & 15 & 100 & 120 & 0 \\
Destination & 150 & 120 & 120 & 180 \\
\hline
\end{tabular}

Figure 7 shows the graph of the servo motors' motion in degrees as it moves from the home position to pick and place the food and glass from their initial locations to the destination. The execution time for the robot's operation has been recorded. In the experiment, the whole task has been achieved within 380 seconds. This duration was dependent on the objects' position and the motors' speed adjusted for faster motion. However, if the robot's joints were moving too fast, such as $0.78 \mathrm{rad} / \mathrm{s}$ could cause the whole robotic arm structure to be unstable, and if its motion were too slow such as 0.078 $\mathrm{rad} / \mathrm{s}$ would be inefficient since the robot would be taking too long to accomplish each task. From the experiments conducted, the success rate of the robotic arm in picking up and placing the object is $70 \%$, where the success rate has been calculated as

Success rate $=\frac{\text { Number of successful trials }}{\text { Total number of trials }} \times 100$

In the unsuccessful attempts, the robotic arm failed to move to the desired positions to pick up or place the objects as required. The wires connected to the Arduino ports tend to lose at times after several operation cycles. In the future, this problem may be improved using another microcontroller or enhancing the electronic connections at the ports. Due to the low cost and simple nature of the robotic arm, the variation of the objects it can carry is limited to $8 \mathrm{~cm}$ in width and 500 grams. However, many of the essential items in daily living activities fall under this category, such as the spoon's food, $300 \mathrm{ml}$ of the glass of water, combs, and toothbrush. Further improvement can be made by incorporating motors with higher torque, but this will incur a higher cost.

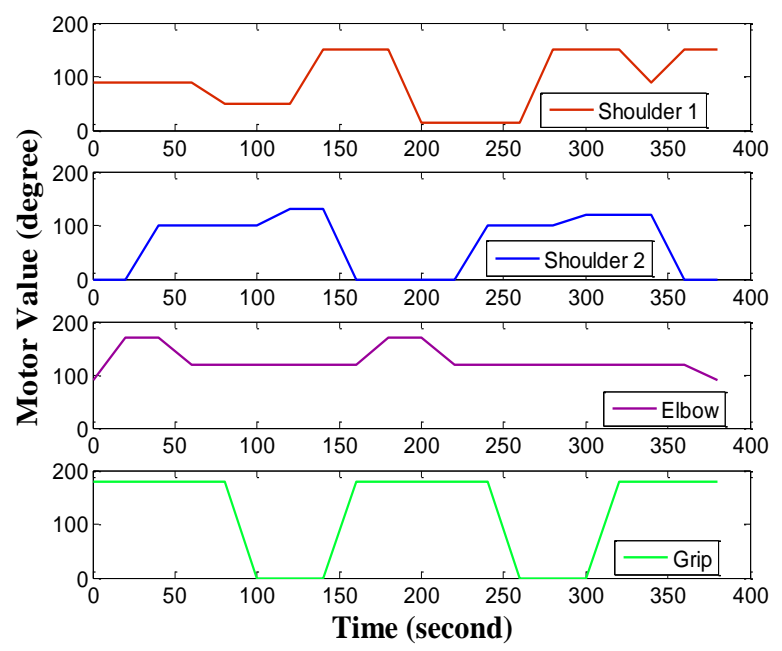

Figure 7. The sequence of the four motors movement during pick and place task. 


\section{CONCLUSION}

A low cost 4-DOF anthropomorphic robotic arm for the disabled and humanoid application has been presented in this paper. The anthropomorphic arm's mechanical design has been made simple, lightweight, and low cost by reducing the number of arm joints while ensuring the desired task's accomplishment and selecting the components. The forward and inverse kinematics analysis of the robot has been conducted to calculate the required each joint's rotation to reach the necessary locations. The results have shown that the proposed low-cost anthropomorphic robotic arm successfully performs the pick and place task for food and glass and puts the objects at the correct position. Future work involves incorporating the robotic system with a camera to automatically determine the object's initial and final locations and developing the control law for precise control of the robot. The artistic features of the robotic arm would also be improved to enhance its anthropomorphic appearance.

\section{CONFLICTS OF INTEREST}

The authors declare no conflicts of interest.

\section{ACKNOWLEDGEMENT}

The authors would like to thank the International Islamic University Malaysia for supporting this research under the research grant number: P-RIGS18-019-0019.

\section{REFERENCES}

[1] D.N. Prabhu, "Kinematic analysis of a 7 DOF anthropomorphic robotic arm having fingers modelled under rigid body mechanics", Master's Theses, Mechatronics Engineering, Politecnico di Torino, Italy, 2018.

[2] N.Z. Azlan and H. Yamaura, "Anthropomorphic finger with optimised geometric parameters for pinching and grasping task", Mechanism and Machine Theory, vol. 49, pp. 52-66, 2012, https://doi.org/10.1016/j.mechmachtheory.2011.1 1.005 .

[3] N.Z. Azlan, and H. Yamaura, "Underactuated anthropomorphic finger mechanism for grasping and pinching with optimised parameter", Journal of Computer Science, vol. 6, no. 8, pp. 928-933, 2010, https://doi.org/10.3844/jcssp.2010.928.933.

[4] S.B. Woo, A. Bodrov, and J. Apsley, "Quasi-optimal energy path planning for anthropomorphic manipulator using gravity torque mapping",
Proceeding 2018 25th International Workshop on Electric Drives: Optimisation in Control of Electric Drives (IWED), Moscow, pp. 1-6, 2018, https://doi.org/10.1109/IWED.2018.8321384.

[5] N. Ibhar, W. Flores, and R. León, "Design of a lowcost teleoperated robotic arm: Assembly and performance testing", Proceeding IEEE 37th Central America and Panama Convention (CONCAPAN XXXVII), Managua, pp. 1-5, 2017, https://doi.org/10.1109/CONCAPAN.2017.82784 90.

[6] J. Sundharsan, and L. Karunamoorth, "Path planning and co-simulation control of 8 DOF anthropomorphic robotic arm", International Journal of Simulation Modelling, vol. 15, no. 2, pp. 302-312, 2016, https://doi.org/ 10.2507/IJSIMM15(2)0.33.

[7] D.S.V. Bandara, R.A.R.C. Gopura, K.T.M.U. Hemapala, and K. Kiguchi, "Development of a multi-DOF trans humeral robotic arm prosthesis", Medical Engineering and Physics, vol 48, pp. 131141,2017 ,

https://doi.org/10.1016/j.medengphy.2017.06.034.

[8] W. Li, P. Chen, Y. Jiang, D. Bai, S. Togo, and H. Yokoi, "Structure design of a tendon-driven robotic arm considering safety and durability", Proceeding 2018 IEEE International Conference on Intelligence and Safety for Robotics (ISR), Shenyang; pp. 71-76, 2018, https://doi.org/10.1109/iisr.2018.8535706.

[9] O. Stan, and A. Mamea, "Design and implement a 6 DOF anthropomorphic robotic-structure", International Journal of Modelling and Optimization, vol. 9, no. 6, pp. 353-356, 2019, https://doi.org/10.7763/ijmo.2019.v9.736.

[10] S. Shen, R. Salvador, M. Eduardo, M. G. Francisco, and $\mathrm{L} . \mathrm{Ni}$, "Development and analysis of robotic arms for humanoid melo", Proceeding ASME 2018 International Mechanical Engineering Congress and Exposition, Pittsburgh, Pennsylvania, usa, pp. 1 -7, 2018, https://doi.org/10.1115/imece2018-87987.

[11] A. Pop, and O. Stan, "Control a 6DOF "Anthropomorphic robotic structure with computer vision as MEMS input", Proceeding 2019 22nd International Conference on Control Systems and Computer Science, Bucharest, Romania, pp. 700-706, 2019, https://doi.org/10.1109/cscs.2019.00125.

[12] B. Liao, H. Zang, N. Zhu, D. Liu, J. Tuo, T. He, L. $\mathrm{Hu}$, and Z. Yang, "System design and experiment of bionics robotic arm with humanoid characteristics", Proceeding 2018 WRC Symposium on Advanced Robotics and Automation (WRC SARA), Beijing, 2018; pp. 90-95, https://doi.org/10.1109/WRCSARA.2018.8584199. 
[13] R. Oliveira, R. Resende, F. Soares, A. Calado, and P. Leite, "Mimicking human movement with robots: control of an anthropomorphic robotic arm using a glove-based system as an educational tool", Proceeding of the 6th International Conference on Control, Decision and Information Technologies (CoDIT), Paris, France, pp. 79-84, 2019, https://doi.org/10.1109/CoDIT.2019.8820516.

[14] J. Grasshoff, L. Hansen, I. Kuhlemann, and K. Ehlers, "7DOF hand and arm tracking for teleoperation of anthropomorphic robots", Proceeding of ISR 2016: 47st International Symposium on Robotics, Munich, Germany, pp. 1-8, 2016, https://doi.org/10.3390/s20113082.

[15] I. Xygonakis, N. Pandria, P. Kartsidis, G Arfaras, K. R. Kavazidi, N. Foroglou, A. Astaras, and P.D. Bamidis, "Towards rehabilitation robotics: Off-theshelf BCI control of anthropomorphic robotic arms", BioMed Research International, pp. 1-17, 2017, https://doi.org/10.1155/2017/5708937.

[16] M.E. Eschenbach, B. Manela, J. Peters, and A. Biess, "Metric-based imitation learning between two dissimilar anthropomorphic robotic arms", arXiv preprint, https://arxiv.org/abs/2003.02638.

[17] A. Sánchez., A. Teran, A. Ibarra, L. Abatta, D. Alulema, D. Morocho, and F. Encalada, "Design and construction of an anthropomorphic robotic arm of seven degrees of freedom with kinematic and dynamic analysis based on genetic algorithms", Proceeding 2016 IEEE International Conference on Automatica (ICA-ACCA), Curico, pp. 1-8, 2016. https://doi.org/10.1109/ICA-ACCA.2016.7778502.
[18] J. Zhao, Z. Feng, F. Chu, and N. Ma, "Advanced theory of constraint and motion analysis for robot mechanisms", The 1st edition, Academic Press, 2014, https://doi.org/10.1016/B978-0-12-4201620.00015-1.

[19] K.H. Chang, "Computer-aided engineering design" The 1st edition, Academic, 2015.

[20] J. Wittenburg, "Kinematics: Theory and applications", The 1st edition, Springer, 2017.

[21] S.B. Niku, "Introduction to robotics: Analysis, control, applications", The 2nd edition, Wiley, 2010 .

[22] T.P. Singh, P. Suresh, and S. Chandan, "Forward and inverse kinematic analysis of robotic manipulators", International Research Journal of Engineering and Technology, vol. 4, no. 2, pp. 14591469, 2017.

[23] M.G. Krishnan, and S. Ashok, "Kinematic analysis and validation of an industrial robot manipulator", Proceeding of IEEE Region 10 Conference (TENCON), Kochi, India, pp. 1393-1399, 2019. https://doi.org/10.1109/tencon.2019.8929712. 